\title{
latrogenic perforation of esophagus successfully treated with Endoscopic Vacuum Therapy (EVT)
}

Authors

Institutions
Gunnar Loske', Tobias Schorsch', Christian Dahm¹, Eckhard Martens², Christian Müller ${ }^{1}$

Department for General, Abdominal, Thoracic and Vascular Surgery, Katholisches Marienkrankenhaus Hamburg gGmbH Department for Medical Oncology and Haematology, Gastroenterology and Infectious Diseases, Katholisches Marienkrankenhaus Hamburg gGmbH
Bibliography

DOI http://dx.doi.org/

10.1055/s-0034-1392566

Published online: 3.8.2015

Endoscopy International Open

2015; 03: E547-E551

(c) Georg Thieme Verlag KG

Stuttgart · New York

E-ISSN 2196-9736

\section{Corresponding author}

\section{Gunnar Loske, MD}

Department for General, Abdominal, Thoracic and Vascular Surgery

Katholisches

Marienkrankenhaus Hamburg

gGmbH

Alfredstrasse 9

22087 Hamburg

Phone: 00494025461402

Fax: 00494025461400

loske.chir@marienkrankenhaus. org
Background and study aims: Endoscopic Vacuum Therapy (EVT) has been reported as a novel treatment option for esophageal leakage. We present our results in the treatment of iatrogenic perforation with EVT in a case series of 10 patients.

Patients and methods: An open pore polyurethane drainage was placed either intracavitary through the perforation defect or intraluminal covering the defect zone. Application of vacuum suction with an electronic device (continuous negative pressure, $-125 \mathrm{mmHg}$ ) resulted in defect closure and internal drainage.

\section{Introduction \\ $\nabla$}

Today, most iatrogenic esophageal perforations are caused by interventional endoscopy. This major complication is associated with significant rates of morbidity and mortality. Operative treatment uses full-scale surgery up to cervical diversion. Conservative therapy ranges from parenteral nutrition, gastric drainage, and antibiotics to interventional techniques such as implantation of covered stents and closure with clips. Esophageal stenting or clipping of esophageal leaks often has to be combined with simultaneous external drainage $[1,2]$.

Endoscopic vacuum therapy (EVT) has been reported as a novel treatment option for esophageal leakage $[3,4]$. Most retrospective studies of the technology summarize heterogeneous cases of anastomotic insufficiency and spontaneous and iatrogenic perforation [5-11]. We gained experience with EVT since 2005 and the aim of this paper is to present our results in treatment of iatrogenic perforation of the esophagus in a series of 10 patients.
Results: Esophageal perforations were located from the cricopharyngeus $(4 / 10)$ to the esophagogastric junction (2/10). EVT was feasible in all patients. Eight patients were treated with intraluminal EVT, one with intracavitary EVT, and one with both types of treatments. All perforations $(100 \%)$ were healed in within a median of $(3-7)$ days. No stenosis occurred, no complications were observed, and no additional operative treatment was necessary.

Conclusions: Our study suggests that intraluminal EVT will play an important role in endoscopic management of esophageal perforation.

\section{Patients and methods}

Between 2007 and 2014, 10 patients from our clinic were found to have iatrogenic perforation caused by endoscopic procedures ( 7 male/ 3 females), aged 28 to 82 years. Since 2007, all cases of iatrogenic esophageal perforation have been treated with EVT.

Open pore drainage consisting of a gastric tube and open pore polyurethane foam (V.A.C. ${ }^{\circledR}$ GranuFoam $^{\mathrm{TM}}$, Kinectic Concepts, Inc., San Antonio, USA; Endo-SPONGETM, B. Braun Melsungen AG, Melsungen, Germany; Suprasorb ${ }^{\circledR}$ CNP Wundschaum, Lohmann\&Rauscher GmbH\&Co. KG, Neuwied, Germany) was used for therapy. The foam fixed at the tip of the tube was placed solely by endoscopic means with a grasper $(\bullet$ Fig. 1 a, - Fig. 2a). The open pore foam adheres to the tissue when connected with an electronic vacuum device (KCI V.A.C. Freedome ${ }^{\circledR}$, KCI USA Inc., San Antonio, Texas, USA, setting: $-125 \mathrm{mmHg}$, continuous, intensity 10). Intraluminal (IL) and intracavitary (IC) variants of therapy have been described previously in detail [12]. Both variants were applied.

Endoscopies were done with $\mathrm{CO}_{2}$ insufflation and standard gastroscopes. 

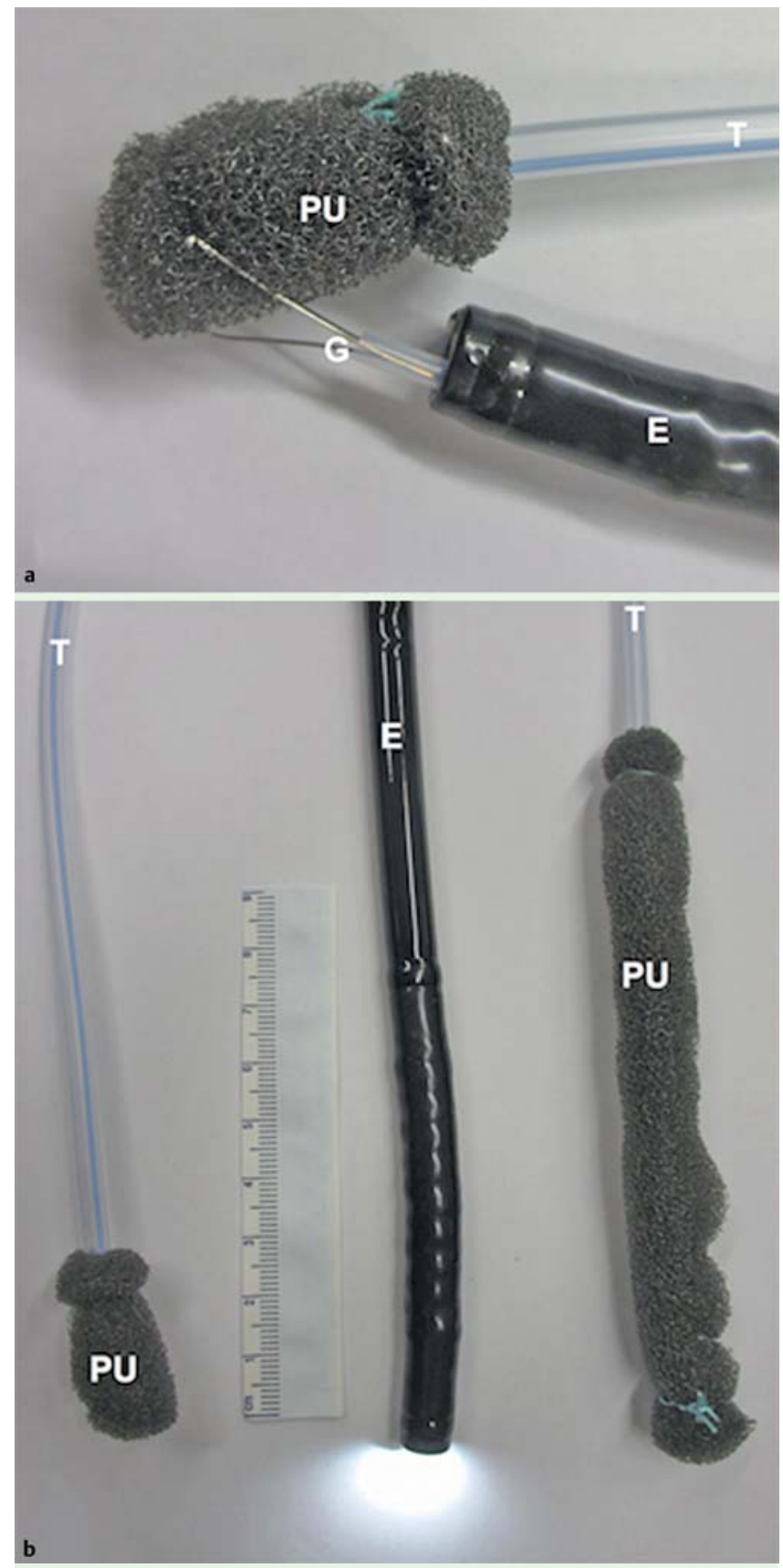

Fig. 1 a Open pore polyurethane foam (PU) fixed at tip of drainage tube $(T)$, endoscope (E), grasper (G), b Open pore drainage. Left: short polyurethane foam (PU) for intracavitary EVT; right: long PU for intraluminal EVT; endoscope (E); drainage tube $(\mathrm{T})$.

Treatment started immediately after the endoscopic diagnosis. Once the perforation defect was passable without endoscopic dilation, intracavitary therapy was used. Otherwise, the open pore foam was placed directly intraluminally onto the epithelium of the esophagus covering the perforation area. Intraluminal EVT resulted in occlusion of the esophageal lumen ( $\bullet$ Fig.3). Contamination of the wound with gastric fluids and saliva was eliminated. Wound secretion and edema were drained permanently in the intraluminal direction opposite the negative intrathoracic pressure.

For intraluminal EVT, we used foam that measured of 1.5 to $2 \mathrm{~cm}$ in diameter and up to $12 \mathrm{~cm}$ in length. For intracavitary place- ment, the foam was trimmed individually to an appropriate size ( $\bullet$ Fig. 1 b). The tube exited nasally and, if necessary, a nasolabial suture was placed to prevent dislocation.

Drainage was removed orally in the first 2 to 5 days simply by withdrawing the tube to allow endoscopic inspection of the inner wound. If the area of perforation was free of inflammation and granulation closed or covered the inner wound, the therapy was discontinued. If not, a new open pore drain was placed and therapy continued. If the therapy was discontinued, liquid intake then began. When endoscopic control showed regular intracorporeal wound healing, a patient was started on a soft diet. After completion of vacuum therapy, follow-up endoscopies were performed to monitor the healing process.

\section{Results}

$\nabla$

All perforations were diagnosed endoscopically in median time on the same day or within 24 hours after a perforation event. Treatment began immediately after diagnosis. Esophageal perforations were located from the cricopharyngeus ( 4 out of 10) to the esophagogastric junction (2 out of 10). Esophageal insertion of open pore drainage was possible in any location.

Eight patients were treated with intraluminal EVT (IL), one with intracavitary EVT (IC) and one patient with both types of treatment (conversion from intracavitary to intraluminal therapy) ( Video 1 ).

After removal of the foam, epithelial tissue that had come into contact with it during EVT showed a typical pimpled erosion pattern ( $\bullet$ Fig. $\mathbf{2}$ b, c). Controlled endoscopy showed that this pattern disappeared within a few days ( Fig. 2 d). In some patients, we observed open pores obstructed partly by viscous saliva or slime. Six of the 10 patients were ventilated during the treatment period, four of them only during the placement procedures.

- Table 1 provides and overview of all important data from the case series. Systemic antibiotics were given in eight cases but as shown in the table, there were no ventilation-associated morbidities.

In all patients treated with intraluminal EVT, the wound edges were found to be closed on the first control endoscopy ( $\bullet$ Fig. 2c). In six patients, EVT was terminated at the first endoscopic control following one period of treatment. Overall, 15 placement procedures were completed. Follow-up endoscopies were performed on nine patients (average 200 days). One asymptomatic patient did not consent to follow-up endoscopy.

In all 10 patients (100\%), the perforation leakages were healed in a median of $5(3-7)$ days after administration of EVT.

No additional operative treatment was necessary. No external thoracic drainage was needed. No extra endoscopic intervention (clip, stent) or other surgical procedures were necessary. No stenosis occurred and no patient complained of dysphagia symptoms after therapy. The healing process was closely monitored by control endoscopy; a second cycle of EVT was not necessary in any of the patients.

\section{Discussion}

$\nabla$

In contrast to anastomotic insufficiency, sudden transmural injury results in a localized inflammation in cases of early iatrogenic perforation. But if saliva, gastric, and choline secretions or infective fluids are brought into continuous contact with extra- 


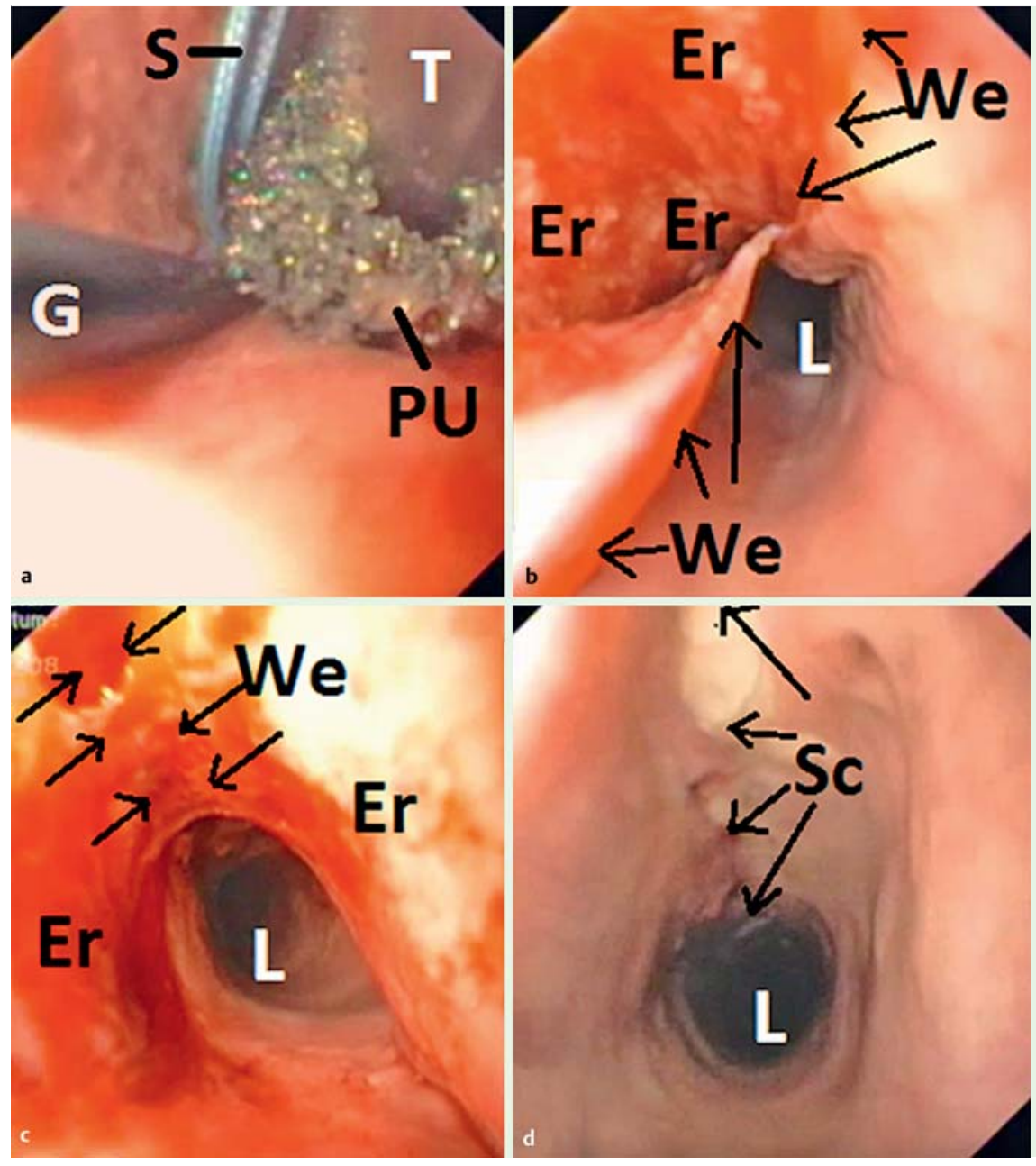

Fig. 2 a Esophageal placement of open pore drainage (polyurethane foam [PU], suture [S], drainage tube $[T]$, grasper [G]. $\mathbf{b}$ After 2 days of intracavitary EVT (wound edges [We] of perforation defect). c After 3 days of intraluminal EVT (wound edges [We] stick together). $\mathbf{d} 8$ days after a 5-day treatment with EVT (erosion pattern has disappeared and a tiny scar $[S c]$ is the residuum of the perforation defect [esophageal Lumen [L]]]).

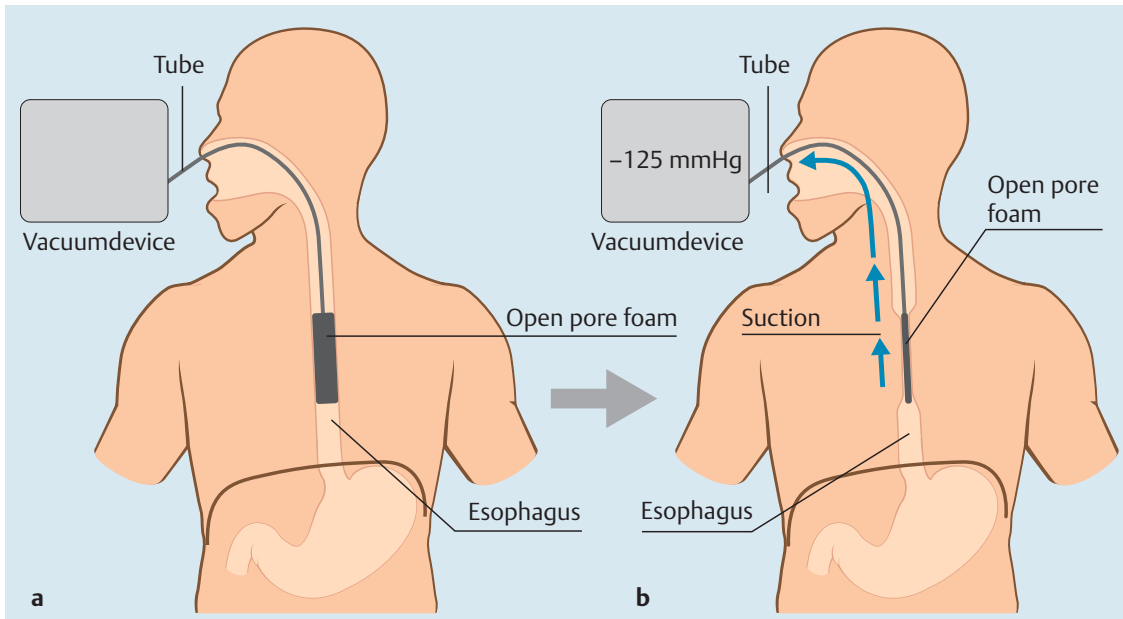

Fig. 3 Schematic for intraluminal EVT. a Open pore foam drainage has been inserted into the esophageal lumen. $\mathbf{b}$ After application of vacuum suction, the esophageal lumen collapses around and with the open pore foam.

luminal tissue through the perforation due to the physiologic intrathoracic negative pressure, development of mediastinitis can be promoted.

In our limited series, we were able to start EVT immediately after diagnosis within 24 hours after a perforation event. Early onset of treatment is known to be a major factor in therapeutic outcome. Recent studies with EVT show a success rate of $85 \%$ to $95 \%$ for use of the approach to treat esophageal anastomotic insufficiencies and perforations in more than 120 patients [7-12].

Endoscopic stenting for sealing esophageal leaks is an established method with over 20 years of clinical experience. However, in six of our patients, perforations were located either ciropharyngeal or near the esophagogastric junction. In those locations, limitations of stent therapy have been described, due to dislocation, migration, persisting leakage and foreign body sensation [13]. We suppose that stenting would not have been an optimal treatment in these cases. To date, two studies comparing stenting with EVT both have suggested that EVT has an advantage over use of stents in terms of outcomes for sealing esophageal leaks $[9,10]$.

Few published reports exist about experience with closure of early-detected esophageal perforation use the over-the-scope-clip 
Table 1 Clinical data on EVT for iatrogenic esophageal perforation.

\begin{tabular}{|c|c|c|c|c|c|c|c|c|c|}
\hline Patient & Origin of defect & $\begin{array}{l}\text { Location from } \\
\text { dental arch }(\mathrm{cm})\end{array}$ & $\begin{array}{l}\text { Defect } \\
\text { size }(\mathrm{mm})^{1}\end{array}$ & $\begin{array}{l}\text { Placement } \\
\text { maneuvers (n) }\end{array}$ & $\begin{array}{l}\text { Days of } \\
\text { EVT (d) }\end{array}$ & $\begin{array}{l}\text { Vacuum } \\
\text { therapy }\end{array}$ & $\begin{array}{l}\text { Days of Ven- } \\
\text { tilation (d) }\end{array}$ & $\begin{array}{l}\text { Antibiotic } \\
\text { (yes/no) }\end{array}$ & $\begin{array}{l}\text { Follow } \\
\text { up (d) }\end{array}$ \\
\hline A & Dilation maneuver & 15 & 30 & 2 & 5 & $\mathrm{IC} / \mathrm{IL}$ & 6 & $y$ & 320 \\
\hline B & Rigid endoscopy & 15 & 10 & 3 & 7 & IL & 8 & $y$ & 9 \\
\hline C & Flexible endoscopy & 14 & 30 & 1 & 7 & IL & invalid $^{3}$ & $y$ & 9 \\
\hline D & Rigid endoscopy & 15 & 15 & 1 & 4 & IL & 1 & $n$ & 7 \\
\hline $\mathrm{E}$ & Dilation maneuver & 25 & 20 & 1 & 3 & IL & 3 & $y$ & 70 \\
\hline $\mathrm{F}$ & Extraction meat bolus & 30 & 10 & 1 & 4 & IL & 1 & $y$ & 67 \\
\hline G & Extraction foreign body & 33 & 50 & 1 & 5 & IL & 5 & $y$ & 18 \\
\hline $\mathrm{H}$ & Dilation maneuver & 35 & 10 & 2 & 7 & IC & 1 & $\mathrm{n}$ & 90 \\
\hline I & Dilation maneuver & 37 & 5 & 2 & 6 & IL & 1 & $y$ & 240 \\
\hline J & Flexible endoscopy & 40 & 10 & 1 & 4 & IL & 2 & $y$ & 4 \\
\hline
\end{tabular}

${ }^{1}$ No dilation of defects

2 No ventilation-associated problems

3 Surgical therapy of ulcus ventriculi bleeding

${ }^{4}$ Clinically asymptomatic patient did not consent to follow-up endoscopy.

method. The procedure seems to be successful but also appears to be associated with considerable risks [14].

We suspect that our short treatment duration (median 5 days) and success rate of $100 \%$ also depends on the parameters of the negative pressure we use. Two technical prerequisites exist for EVT: open pore drainage and negative pressure. Based on our experience with the technology, we believe that it is important to ensure the best suction effect and adherence of the foam within tissue -specific parameters. Permanent suction works with multiple open pores, even if some of them may be obstructed. Since we began performing EVT on the esophagus in 2006, we have continued to successfully use the same electronic device. Current vacuum devices generate negative pressure adapted to specific benefits for superficial wounds. We assume that these modern devices may be not suitable for EVT in the esophagus.

In 2008, we performed our first intraluminal EVT on a patient with cervical iatrogenic perforation. Intraluminal vacuum application resulted in occlusion of the esophagus lumen and contraction of adjacent tissue. The wound edges stuck together and incorporation of septic fluid was avoided. The procedure was easy

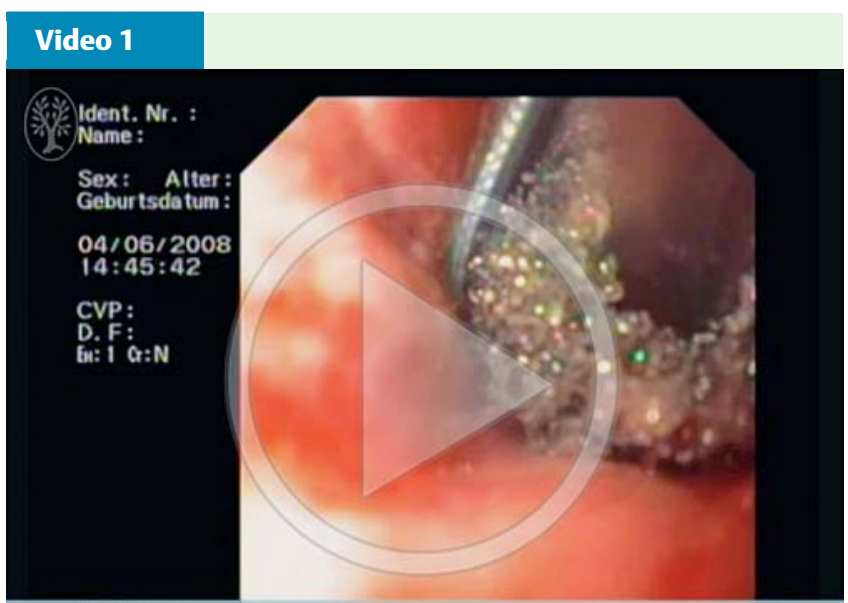

intracavitary EVT, placement of foam drainage

Online content including video sequences viewable at: http://dx.doi.org/ 10.1055/s-0034-1392566 to perform and feasible in nine of 10 patients with iatrogenic perforation.

We have observed that swallowing viscous saliva can result in blockage of open pores in the foam. Our observations suggest, however, that the blockage was not complete and that drainage was still effective. However, blockage of the foam may increase risk that the vacuum will be ineffective. Therefore, we recommend routine endoscopic examination of patients undergoing EVT 3 days after the procedure or in case of any irregular symptoms.

Surgical monitoring following EVT is the same as after any other superficial surgical procedure: Inspection of the wound is necessary to supervise wound healing.

Six of the patients in our series were ventilated during the treatment period to avoid swallowing of saliva and to ensure effective suction of tissue. This might be one of the disadvantages of EVT, yet in our series, we did not observe any comorbidities caused by the short periods of ventilation.

Because the duration of treatment with EVT was short, all of the patients in our series received parenteral nutrition. In cases of anastomotic insufficiency, because the treatment duration was significantly longer, we use adapted feeding tubes [5].

Because none of the patients required external thoracic drainage, it appears that by combining evacuation and a sealing effect, EVT resulted in sufficient drainage in all cases.

The advantages of EVT are its easy application throughout the whole length of the esophagus from the cricopharyngeus to the esophagogastric junction and the ability to simultaneously close and promote internal drainage in one endoscopic step. The treatment period is short and no complications have been observed. Our study suggests that EVT will play an important role in endoscopic management of all types of esophageal perforation. Additional studies would be of interest to further prove its effectiveness under various conditions.

Competing interests: Gunnar Loske is a consultant for Lohmann \& Rauscher GmbH \& Co. KG. Tobias Schorsch, Christian Dahm, Eckhard Martens and Christian Müller declare that no conflict of interest exists. 


\section{References}

1 Carrott PWJr, Low DE. Advances in the management of esophageal perforation. Thorac Surg Clin 2011; 21: 541 - 555

2 Schmidt SC, Strauch S, Rosch T et al. Management of esophageal perforations. Surg Endosc 2010; 24: 2809-2813

3 Mennigen R, Senninger N, Laukoetter MG. Novel treatment options for perforations of the upper gastrointestinal tract: endoscopic vacuum therapy and over-the-scope clips. World J Gastroenterol 2014; 20 : $7767-7776$

4 Schaheen L, Blackmon SH, Nason KS. Optimal approach to the management of intrathoracic esophageal leak following esophagectomy: a systematic review. Am J Surg 2014; 208: 536-543

5 Schorsch T, Muller C, Loske G. Endoscopic vacuum therapy of anastomotic leakage and iatrogenic perforation in the esophagus. Surg Endosc 2013; 27: 2040-2045

6 Kuehn F, Schiffmann L, Rau BM et al. Surgical endoscopic vacuum therapy for anastomotic leakage and perforation of the upper gastrointestinal tract. J Gastrointest Surg 2012; 16: 2145-2150

7 Weidenhagen R, Hartl WH, Gruetzner KU et al. Anastomotic leakage after esophageal resection: new treatment options by endoluminal vacuum therapy. Ann Thorac Surg 2010; 90: 1674-1681
8 Heits $N$, Stapel L, Reichert B et al. Endoscopic Endoluminal Vacuum Therapy in Esophageal Perforation. Ann Thorac Surg 2014; 97: 1029 1035

9 Schniewind B, Schafmayer C, Voehrs $G$ et al. Endoscopic endoluminal vacuum therapy is superior to other regimens in managing anastomotic leakage after esophagectomy: a comparative retrospective study Surg Endosc 2013; 27: 3883 - 3890

10 Brangewitz M, Voigtlander T, Helfritz FA et al. Endoscopic closure of esophageal intrathoracic leaks: stent versus endoscopic vacuumassisted closure, a retrospective analysis. Endoscopy 2013; 45: 433 438

11 Bludau M, Holscher AH, Herbold T et al. Management of upper intestinal leaks using an endoscopic vacuum-assisted closure system (E-VAC). Surg Endosc 2014; 28: 896-901

12 Loske G, Schorsch T, Muller C. Intraluminal and intracavitary vacuum therapy for esophageal leakage: a new endoscopic minimally invasive approach. Endoscopy 2011; 43: 540-544

13 Voermans RP, Ponchon T, Schumacher B et al. Forward-viewing versus oblique-viewing echoendoscopes in transluminal drainage of pancreatic fluid collections: a multicenter, randomized, controlled trial. Gastrointest Endosc 2011; 74: 1285-1293

14 D'Cunha J, Rueth NM, Groth SS et al. Esophageal stents for anastomotic leaks and perforations. J Thorac Cardiovasc Surg 2011; 142: 39-46 